Instituto Internacional de Investigación y Desarrollo Tecnológico Educativo INDTEC, C.A.

DOI: https://doi.org/10.29394/scientific.issn.2542-2987.2017.2.3.10.190-208

OAI-PMH: http://www.indteca.com/ojs/index.php/Revista Scientific/oai

\title{
Estrategias Formativas de Sensibilización para el Tratamiento de los Desechos Tecnológicos Informáticos en Instalaciones de Barinas I UNELLEZ VPDS Barinas 2016
}

\author{
Autora: Heiddy Ludmila Lizcano de Aviles \\ Universidad Pedagógica Experimental Libertador, UPEL \\ kemyleo@gmail.com \\ Barinas, Venezuela
}

\section{Resumen}

A través de la presente investigación se propone Evaluar las Estrategias Formativas de sensibilización para el Tratamiento de los Desechos Tecnológicos Informáticos en las Instalaciones de Barinas I del VPDS UNELLEZ Barinas año 2016, esto debido a la acumulación desorbitada que se está presentando en las instalaciones de la universidad ante la ausencia de información sobre la contaminación que estos residuos generan, la modalidad es de proyecto factible, la naturaleza de la investigación es cuantitativa, de tipo descriptiva de campo, los datos arrojados en la aplicación de las estrategias serán a partir del conocimiento y experiencias de los trabajadores abordados con el fin de involucrarlos en la solución de esa problemática ambiental. La población está definida por (55) trabajadores de las áreas de Barinas I, la muestra será estratificada comprendida por (17) individuos. Los instrumentos a emplear serán la observación y el cuestionario tipo encuesta comprendido en (12) ítems. Todo con el objeto de sensibilizarlos ante la protección y preservación necesaria para disminuir este tipo de contaminación que está presente en las aéreas físicas de la universidad como garantía de una mejor calidad de vida presente y futura de la población, por medio de procesos metodológicos que impliquen planificar actividades que permitan una mejor consecución de los objetivos.

Palabras clave: estrategias formativas; sensibilización; desechos tecnológicos informáticos.

Fecha de Recepción: 13-08-2016

Fecha de Aceptación: 19-09-2016 


\title{
Awareness Training Strategies for Treatment of Information Technology in Waste Facilities Barinas I UNELLEZ VPDS Barinas 2016
}

\begin{abstract}
Through the present research is proposes evaluate them strategies training of awareness for the treatment of them waste technological (computer) in the facilities of Barinas I of the VPDS-UNELLEZ Barinas year 2016, this due to it accumulation exorbitant that is presenting in them facilities of the University before it absence of information on the pollution that these waste generated, it mode is of project feasible, it nature of it research is quantitative, of type descriptive of field, the data thrown in it application of them strategies will be starting from the knowledge and experiences of them workers addressed to involve them in it solution of that problematic environmental. The population is defined by (55) workers in the areas of Barinas I, sample will be stratified by (17) individuals. Those instruments to use will be the observation and the questionnaire type survey covered in (12) items. All to raise them before the protection and preservation required for decrease this type of pollution that is present in them air physical of the University as warranty of a best quality of life present and future of the population, by means of processes methodological that involve plan activities that allow a best achievement of them objectives.
\end{abstract}

Keywords: training strategies; awareness-raising; technological computer waste.

Date Received: $13-08-2016$

Date Acceptance: 19-09-2016 


\section{Introducción}

En la actualidad, la evolución tecnológica ha contribuido a aumentar la diversidad y complejidad de los desechos que contaminan el medio ambiente, el gran crecimiento en la producción de aparatos eléctricos que gracias a la innovación tecnológica y la globalización del mercado aceleran su sustitución y por lo tanto sus desechos lo que produce diariamente toneladas de basura electrónicos informáticos.

La era tecnológica nos ha brindado maravillas que sólo se encontraban en nuestros sueños hace algunos años, pero todas estas ventajas también tienen sus efectos negativos, uno de ellos es el desperdicio electrónico; el recambio informático es tan rápido y frecuente que una enorme cantidad de dispositivos se vuelven obsoletos en tiempo récord y terminan su existencia en un basurero.

Lamentablemente, en la mayoría de los países la basura electrónica informática es tratada de la misma manera que la basura convencional y termina siendo arrojada en lugares inadecuados para ello, cualquier persona puede pensar que una placa expuesta al ambiente no es del todo perjudicial, pero nada está más lejos de la verdad. En componentes electrónicos descartados es posible encontrar elementos peligrosos, colocar este tipo de residuos en la basura o dejarlos en manos de cartoneros, es poner en riesgo la salud de las personas y el deterioro, degradación y contaminación del ambiente, debido a que contienen componentes peligrosos.

Mientras el celular, el monitor, el CPU, los teclados, las impresoras y el televisor están en su casa no generan riesgos de contaminación, pero cuando se mezclan con el resto de la basura y se rompen esos metales tóxicos se desprenden y pueden resultar mortales, la mayoría de las personas no toman conciencia sobre lo que está sucediendo con nuestro mundo, la tecnología desde la perspectiva de la informática llámense computadores, laptop, tabletas, impresoras, digitalizadores de imágenes y documentos; además de 
otros objetos que comprenden esta rama fueron creados por el hombre con el objeto de facilitarse el trabajo de manejo, almacenamiento y recolección de información de tipos cualitativo y cuantitativo entre otros, no obstante esta innovación ha ido evolucionado al pasar de los años rebasando todas las necesidades del individuo en sus diferentes ámbitos laborales y cotidianos exigiéndole a su vez la adaptación y adquisición de herramientas tecnológicas-informáticas más avanzadas.

En muchos países aún no se le da la importancia a la separación de la basura; considerando que la gente misma no sabe diferenciar los desechos orgánicos de los inorgánicos y ahora que se pretende separar también los desechos tecnológicos informáticos; la preocupación va en aumento, puesto que las personas no tienen conocimiento del impacto al medio ambiente que tienen sobre todo el "e-waste" o basura electrónica.

Un sin número de naciones representan en la actualidad grandes "cybertederos" de desechos computacionales y electrónicos, los tratados que tienen como objetivo optimizar o modificar el modo de producción y los elementos utilizados para fabricar los equipos electrónicos no son aprobados por quienes se suponen son los más grandes contaminantes. Según estudio realizado por Amos Unshelm Báez (2011) denominado "Los Desechos Electrónicos, La Basura del Siglo XXI Una Realidad Ambiental MISREDES Mérida, Venezuela, señala que:

Una gestión ambiental tecnológica eficiente debe identificar sus elementos funcionales que son las Fuentes de Generación, las Técnicas de Recuperación y Procesamiento, los Métodos de Tratamiento y Disposición Final y las Formas de Almacenamiento, así como los Medios de Recolección y Transporte (pág. 9).

En síntesis, el autor expresa que cuando los aparatos electrónicos son desechados junto con los demás residuos, previamente se debe tener conocimiento acerca de la clasificación de sus partes ya que la humedad y la 
temperatura ambiental hacen que los componentes altamente tóxicos como el plomo, cadmio, arsénico, mercurio, selenio, etc. Empiecen a fundirse, desintegrarse y liberar las sustancias al medio ambiente afectando el suelo, el agua y el aire.

Por otra parte, la Organización de las Naciones Unidas para la Educación, la Ciencia y la Cultura (UNESCO, 2010), (RELAC) Los Residuos Electrónicos: Un desafío para I sociedad del conocimiento en América Latina y el Caribe indica que: “...los equipos electrónicos contienen hasta 17 metales preciosos incluyendo oro, plata y cobre, los cuales siguen teniendo un valor económico significativo cuando los aparatos caen en desuso" (pág. 08). queriendo referirse además que éstos elementos químicos como "gemas preciosas", son extraídas de las chatarras electrónicas por los pepenadores o recuperadores y que se ha convertido hoy en un gran negocio global con sus características lucrativas; sin dejar de apreciar con seriedad la responsabilidad social que ocasionan en la salud de quienes originalmente extraen dichos componentes, y menos aún los problemas ambientales posteriores que acarrean sobre el entorno, donde en muchos casos utilizan la incineración para tales fines y los que determinen las técnicas del reciclaje de los mismos.

Venezuela no está exenta de padecer esta realidad de contaminación silenciosa que se está desarrollando por los desechos tecnológicos informáticos, tomando en cuenta que por encontrarnos dentro de la clasificación de países en vías de desarrollo intentamos día a día mantenernos actualizados en esta área de la computación, por ende debido a este fenómeno de los avances tecnológicos al año se generan cientos de toneladas de desechos informáticos que no tienen el tratamiento especial necesario para reciclarlos.

En la administración pública el estado venezolano debe implementar una serie de programas los cuales se obligan a estar formados por diferentes proyectos que son sumamente importantes para el desarrollo de la misma, 
donde llevan una fase de ejecución que encamine estas instituciones a una serie de metas trazadas para el mejor avance los mismos.

El vertiginoso avance de la tecnología está provocando que los productos informáticos en cuestión de meses se vuelvan obsoletos 0 incompatibles, o tal vez sigan sirviendo pero por diversos factores como la moda, la innovación, la novedad o el qué dirán, nos lleva a obtener los productos que acaban de sacar al mercado a pesar de que con el anterior se podía trabajar sin ningún problema, esto ha sido evidencia a través de la experiencia de la autora en su desempeño laboral docente en la Universidad Nacional Experimental de los Llanos Occidentales Ezequiel Zamora (UNELLEZ) VPDS Barinas.

Por ende, es ahí donde radica el presente artículo científico, en la investigación de los aparatos que se dejan de utilizar porque se cuenta con la adquisición de otros más actualizados y más nuevo, ya que después de dejarlos un tiempo guardados en donde menos nos estorbe optamos por tirarlos a la basura o vertederos inadecuados de la universidad sin darnos cuenta todo el mal que causamos a nosotros mismos y a las instalaciones de la universidad y al ambiente.

Es importante resaltar que la Administración Pública Nacional requiere de procesos administrativos eficientes para la adquisición de hardware y software, como lo respalda la Ley de Infogobierno (2013) en el Capítulo II Principios y bases del uso de las tecnologías de información, Obligatoriedad del uso de las tecnologías de información, Artículo 15 el cual describe:

En el diseño y desarrollo de los sistemas, programas, equipos y servicios basados en tecnologías de información, se debe prever las consideraciones de accesibilidad y usabilidad necesarias para que estos puedan ser utilizados de forma universal por aquellas personas que, por razones de discapacidad, edad, o cualquier otra condición de vulnerabilidad, requieran de diferentes tipos de soportes 0 canales de información (págs. 6-7). 
Es decir, desarrollar procesos metodológicos que impliquen planificar actividades que permitan una mejor consecución de los objetivos. Por ende, es necesario contar con niveles de planificación y organización a manera de determinar el conocimiento público que cada organismo de la Administración Pública Nacional realice una planificación estratégica que abarque la necesidad de las instituciones educativas universitarias en lo que se refiere a la compra de equipos computacionales, o adquisición del hardware, y que asimismo establezcan normas y procedimientos para su uso.

Es por esto que las estrategias formativas de sensibilización que se van a aplicar ayudarán a generar la sensibilización necesaria para disminuir este tipo de contaminación que está presente en la Universidad Nacional Experimental de los Llanos Occidentales Ezequiel Zamora UNELLEZ Barinas específicamente en las instalaciones de Barinas I incentivando así a los trabajadores a darle la acorde distribución que estos reciclajes de equipos informáticos exigen.

La ciudad de Barinas no escapa a esta realidad ambiental sobre el manejo integral y sostenible de los residuos y desechos electrónicos., debido a que igual que en el resto del país y de la mayoría de los países del mundo, los componentes, aparatos, artefactos, equipos, piezas y accesorios electrónicos, y sus materiales peligrosos, convergen con el resto de los residuos y desechos sólidos municipales, en la misma ruta, desde la mismas fuentes de generación hasta sus lugares de disposición final.

Es por ello, que se debe impulsar, fortalecer y consolidar una política de sensibilización ambiental que pueda integrar a todos los actores sociales en esta delicada materia. En referencia a todo lo antes planteado y a los fines de concretar la situación del problema a estudiar se formulan las siguientes interrogantes: 
¿Cuál es la situación de contaminación que se vive en las instalaciones de Barinas I de la UNELLEZ VPDS ocasionada por los desechos tecnológicos informáticos?

¿Cuál es la factibilidad socioeducativa en la creación de estrategias formativas de sensibilización que conduzcan a la preservación de las instalaciones de Barinas I de la UNELLEZ VPDS?

¿Cuáles las estrategias formativas que tengan como objeto $\mathrm{El}$ Tratamiento de Los Desechos Tecnológicos Informáticos en las Instalaciones de Barinas I de la UNELLEZ VPDS Barinas 2016

¿Cuál es el proceso para la aplicación de estrategias formativas que conduzca a la sensibilización ambiental en el uso de los desechos tecnológicos informáticos en las instalaciones de Barinas I de la UNELLEZ VPDS?

Es importante mencionar que la presente investigación se sustenta bajo la línea de investigación de educación, desarrollo y gestión comunitaria exigida por la UPEL.

\subsection{Objetivo de la Investigación}

Evaluar la aplicación de Estrategias Formativas de sensibilización para el Tratamiento de los Desechos Tecnológicos Informáticos en las Instalaciones de Barinas I de la UNELLEZ VPDS 2016.

\section{Teoría y Conceptos}

\subsection{Antecedentes de la Investigación}

Hidalgo (2013) en su trabajo de investigación de Gestión ambiental denominado "La Basura Electrónica y la Contaminación Ambiental", tuvo como objetivo fundamental identificar a los principales aparatos eléctricos y electrónicos, sus componentes peligrosos para el medio ambiente y para el ser humano. Donde aplico la metodología de recopilación bibliográfica donde 
busca conocer la situación actual de los principales aparatos electrónicos que mayormente están siendo utilizados por la humanidad señalando que:

Los equipos informáticos su vida útil o de renovación, su composición e identificación, las formas de deshacerse y sus componentes son los elementos que atentan contra el medio ambiente y el ser humano, ya que, son las razones por las cuales los vuelven peligrosos (pág. 43).

En síntesis, se expresa que el autor como acción principal elaboró un diagnostico el cual le permitió visualizar la problemática en cuanto contaminación por aparatos electicos y eléctricos causantes estos de acumulación de basura logrando así identificar de forma precisa los diferentes objetos generadores de componentes de alta peligrosidad para el ambiente. Es notorio señalar que esta investigación hace referencia al presente estudio desde la perspectiva de la temática debido a que los desechos electrónicos y sus efectos contaminantes son unas de las causas principales del deterioro y degradación de los suelos, así como factores de riesgos de salud.

Pineda (2012), en su trabajo de investigación titulado: "Modelo para la Gestión de Reciclaje de Residuos Electrónicos de la universidad de Chile Facultad de Ciencias Físicas y Matemáticas Departamento de Ingeniería Eléctrica", por medio de la utilización del método de separación utilizando electromagnetismo expresa que:

Este trabajo busca hacer un aporte en la solución a un problema ecológico, esto es el impacto producido por la acumulación de residuos electrónicos sin tener tratamientos adecuados, en el cual se encuentran en juego factores de tipo económico, ambiental y social.

En conclusión, se puede acotar que el autor señala que los productos electrónicos han pasado a formar parte de las herramientas habituales que se ocupan diariamente ya que se encuentran integradas en los vehículos, en computadoras o aparatos portátiles, electro medicina y sectores de servicios, 
la presente investigación tiene relación con el tema en estudio ya que la temática de los equipos electrónicos informáticos sobre el ambiente a partir de su extracción, manufactura, uso, desecho y descarte, se obvian los métodos de almacenamiento que a largo o corto plazo generan contaminación ambiental.

\subsection{Estrategia Formativa}

Son aquellas habilidades cognitivas que pretenden realizar un análisis tanto de los aspectos referidos al sujeto identificando conductas, conocimientos previos como el objeto general, realizando un análisis de la realidad en la que se va a ejecutar sin perder de vista el objeto final, luego se selecciona y se aplican de manera secuenciada los conocimientos, tal como lo señala Cano (2007):

El desarrollo de una competencia es una actividad cognitiva compleja que exige a la persona establecer relaciones entre la práctica y la teoría; transferir el aprendizaje a diferentes situaciones, aprender a aprender, plantear y resolver problemas y actuar de manera inteligente y crítica en una situación (pág. 39).

Las estrategias formativas como lo compone sus dos palabras son formas o maneras son las prácticas que se ejecutan para desarrollar una competencia cognitiva basado en la teoría y la praxis para impulsar conocimientos, herramientas que permitan aprender a aprender, a resolver situaciones y proporcionar aprendizajes significativos de manera formativa y progresiva.

\subsection{Reciclaje de equipos informáticos}

Cada año salen al mercado nuevas versiones de microprocesadores, teléfonos móviles, scanner, cpu, cd, cámaras, video grabadoras, ordenadores, sistemas operativos, entre otros, los cuales son vendidos remplazando los 
modelos anteriores, el resultado de esta práctica es que cada año se desechan o descartan un sinnúmero de equipos informáticos generando así desechos que pueden ser reciclados como equipos, tal como lo expresa Martínez, Naranjo y Garrido (2007) reciclajes de equipos informáticos en Teruel al desarrollo de un plan de reciclaje de equipos informáticos, con la finalidad de permitir la reutilización de los viejos equipos cedidos por parte de las Administraciones Públicas, empresas, asociaciones o ciudadano.

Es notorio reflejar que el problema o los problemas ambientales que provocan los desechos informáticos serian fácilmente solucionados si se ejecutaran las políticas reciclaje de los diferentes equipos informáticos.

\subsection{Tratamiento de los desechos tecnológicos informáticos}

Los equipos informáticos son desechados a los 3 o 4 años de uso mucho antes del fin de su vida útil, acabando en vertederos o arrojados en contenedores normales, dando pie a que la producción de residuos electrónicos crezca 3 veces más rápido que la medida de los residuos domésticos urbanos. Los materiales más abundantes en un ordenador son plástico, acero, silicio, aluminio, cobre, cadmio, mercurio, cromo, diamante, oro, magnesio entre otros siento algunos de estos muy contaminantes con efectos cancerígenos y que por lo tanto son muy peligrosos al tirar estos desechos en vertederos no actos para el mismo. Así como lo señala Castan (2008):

Se recicla poco el material informático y se manda mucho al tercer mundo que se ha convertido en un enorme vertedero de basura electrónica. La nuestra es una sociedad que lo hace todo con ordenadores y cada vez les pedimos más tareas. El resultado es la generación de miles de toneladas de basura electrónica. Esta velocidad progresiva de obsoletización del material informático ha creado un enorme problema ambiental, porque en la fabricación de ordenadores y electrónica de consumo en general se emplean materiales tóxicos para nuestro entorno... (pág. 3) 
De lo antes expuesto se puede acotar que el flujo de residuos de aparatos electrónicos está creciendo con gran rapidez, estos aparatos contienen grandes cantidades de todo tipo de sustancias peligrosas como metales pesados y sustancias halogenadas, además, se necesitan muchas materias primas para fabricar nuevos aparatos, por lo que se apuesta a la gestión de estos residuos, no siendo destinados a vertederos.

\section{Metodología}

El marco de la investigación se fundamenta en el paradigma cuantitativo que de acuerdo a Sampieri (2006) señala que: "se dedica a recoger, procesar y analizar datos cuantitativos o numéricos sobre variables previamente determinadas (pág. 12), como fundamento a las variables del estudio, la investigación es de proyecto factible, donde el Manual de Trabajos de Grado, de la UPEL (2014) señala que consiste: en la investigación, elaboración y desarrollo de una propuesta de un modelo operativo viable para solucionar problemas, requerimientos o necesidades de organizaciones o grupos sociales y que las mismas deben estar comprendidas por las siguientes fases: diagnostico, diseño de la propuesta y factibilidad de las acciones y estrategias.

De allí, se hace necesario detallar cada una de las fases de la siguiente manera:

Fase I: Diagnóstico: En este se llevará a cabo una evaluación preliminar aplicando la técnica de la observación directa y la encuesta, para ello se aplicará como instrumento de recolección de datos un cuestionario policotómico para conocer la realidad de la temática en estudio, esta primera etapa está considerada como el diagnóstico de la investigación, es por lo tanto, es la recolección cuidadosa y detallada de la realidad en la que se pretende intervenir y la valorar la problemática. 
Fase II: Diseño de la propuesta: El propósito de la misma será elaborar las estrategias formativas de sensibilización en valores sociales ambientales dirigido a los trabajadores de las instalaciones de Barinas I VPDS Barinas que son el área en estudio, la elaboración de la propuesta se realizará de acuerdo a un proceso dinámico, coherente, continuo y orientado a la ejecución de programas donde se refleje la justificación del mismo, contenidos a tratar, tiempo, lugar, recursos y objetivos previamente planteados. (pág. 56).

Fase III: Factibilidad de las acciones y estrategias: Se refiere a la factibilidad de la misma en el espacio geográfico en estudio seleccionado, sustentándose en la colaboración de trabajadores de las instalaciones de Barinas I de la UNELLEZ VPDS seleccionados y en los recursos financieros disponibles de la autora para la realización de la propuesta.

Las fases antes mencionadas son unos de los elementos importantes dentro de esta investigación ya que permitirán la visualización de la problemática de forma más precisa y directa. La presente investigación se enmarca en un diseño descriptivo de campo bajo la modalidad de proyecto factible, el cual según Hernández, Fernández y Baptista (2006) comprende las siguientes fases: diagnostico, diseño, ejecución, evaluación y sistematización, por ende, se hace necesario recorrer cada uno de estas fases con el propósito de obtener los resultados significativos del estudio.

\subsection{Población y muestra}

De igual manera para fundamentar este tópico se señala que según el Manual de Trabajo de Grado Especialización y Maestría y Tesis Doctorales de la UPEL (2014): se entiende como población: "El conjunto finito e infinito de unidades de análisis, individuo u objetos que se someten a estudios; pertenecen a la investigación y son la base fundamental para obtener la información". (pág. 70). 
Por lo tanto, para la presente investigación o estudio la población está constituida por (55) trabajadores que laboran en las instalaciones de Barinas I de la UNELLEZ VPDS y en función a los tipos de muestra existentes y a la escogencia de la más acertada para la presente investigación se utilizara la muestra estratificada la cual según Márquez (2000), en el Manual del proceso de Investigación de las ciencias sociales de la UNELLEZ Barinas consiste en:

Dividir la población en diferentes estratos con el fin de dar representatividad a los distintos factores que integran la población, posteriormente de estos estratos se selecciona una muestra al azar. En cada estrato la representación de los elementos debe ser proporcional a su representación en la población.

Para seleccionar su tamaño, se recomienda según expertos para estudios descriptivos tomar un aproximado del $30 \%$ de la población con un nivel elevado de representatividad, por lo tanto la muestra quedo estructurada por diecisiete trabajadores aplicándole la formula antes mencionada como se indica a continuación: Coordinadores (2), Operadores (5), Técnicos (03), Docentes (4) y Empleados (3).

\subsection{Técnica de recolección de información}

En la presente investigación se pondrá en ejecución la técnica de la encuesta y la observación directa, aclarando así que encuesta según Sampieri (2006): "tiene aplicación en aquellos problemas que se pueden investigar por método de observación, análisis de fuentes documentales y demás sistemas de conocimiento, además permite el conocimiento de las actitudes, opiniones de los individuos con relación al objeto de investigación" (pág. 106).

En síntesis esta técnica se considera pertinente en la investigación ya que permitirá obtener información de la muestra seleccionada, con la finalidad de recopilar todo lo referente a la temática así como por medio de la observación la cual aporte datos en la modalidad escrita que pueden ser 
referentes a la investigación, la cual, Hurtado de Barrera (2010), expresa que: "el investigador tendrá acceso directo al evento de estudio y será testigo de las manifestaciones del evento, se realizara la observación directa en el sitio de investigación u objeto de estudio para recabar la información positiva a la investigación" (pág. 31).

Como instrumento de recolección de datos se utilizará la entrevista donde la misma toma la forma de un interrogatorio en el cual las preguntas se plantean siempre en el mismo orden y además se formulan con los mismos términos. Según Sampieri (2006) expresa que: La entrevista no se considera una conversación normal, sino una conversación formal, con una intencionalidad, que lleva implícitos unos objetivos englobados en una Investigación (pág. 18)

\section{Resultados}

La presente información se sustenta en la aplicación de las estrategias de tipo formativas que fueron pertinentes para obtener información positiva y significativa como fueron: charla, conversatorio y taller informativo en cuanto a la acumulación de desechos tecnológicos informáticos sus causas y consecuencias así como la explicación y discernimientos dentro del área en estudio como lo fue las instalaciones de Barinas I de la UNELLEZ VPDS, se logró recabar datos importantes que permitieron señalar la realidad del caso disertado, por ende, se puede reflejar que la población inmersa en la temática investigada expresaron que la institución universitaria no cuenta con métodos, técnicas, herramientas o políticas internas que proporcionen información acerca del nivel de contaminación que están generando estos desechos tecnológicos en los espacios físicos del pabellón 7 de Barinas I, donde de manera inescrupulosa se almacenan diferentes tipos de objetos electrónicos sin tomar en cuenta su gravedad ambiental. 
Dándole respuesta a la variable sensibilización se pudo notar desde la observación y el registro de información en los momentos de la aplicación de las estrategias que los empleados encargados de estas instalaciones no tienen el menor interés para ayudar en esta problemática ambiental presentes en su área de trabajo y que de una u otra forma a corto, mediano o largo plazo les puede traer consecuencias dermatológicas y pulmonares. $Y$ como finiquito de las variables estos desechos tecnológicos informáticos, la cual arrojo como resultado que los trabajadores y empleados de las instalaciones de Barinas I de la UNELLEZ no tenían el conocimiento del como clasificar los diferentes equipos informáticos desechados por las diferentes áreas administrativas de los departamentos existentes en esta infraestructura.

\section{Conclusiones}

Las conclusiones que arrojan la siguiente temática son claras, las mismas son extraídas de los resultados dando como finiquito que la universidad nacional experimental de los llanos occidentales Ezequiel Zamora (UNELLEZ VPDS) son cuenta con estrategias y políticas públicas que contrarresten o eliminen en su totalidad la acumulación de equipos informáticos en mal estado dentro de las instalaciones de Barinas I ya que no han creado planes de recolección de desechos sólidos que puedan sacar y darle el vertedero necesario para su desecho, por lo tanto se pudo lograr llevarles en diferentes estrategias y modalidad la información a los trabajadores y empleados de manera directa y por diferente permitiendo así generar conocimientos previos y fortalecidos en cuanto a la forma de preservar los diferentes ambiente dentro de la universidad.

\section{Recomendaciones}

Los resultados y conclusiones antes mencionados arrojo para la investigadora las siguientes recomendaciones: 
- Crear estrategias de formación en los temas ambientales.

- Generar políticas y propuestas ante la institución universitaria para erradicar el bote indiscriminado de equipos informáticos.

- Fomentar por medio de actividades participativas la preservación del ambiente.

- Reciclar y reutilizar los equipos desechados en diferentes formas como materos, herramienta de oficina entre otros.

- Impulsar los valores morales, humanistas, y ambientales en los trabajadores, empleados, docentes y estudiantes para proteger los espacios e infraestructuras de la universidad.

\section{Referencias}

Amos, U. (2011). Los Desechos Electrónicos, La Basura del Siglo XXI. Mérida, Venezuela: MISREDES. Recuperado de: http://misredes.com.ve/pdf doc/notas/notas pdf/nota 1.pdf

Cano, E. (2007). Las competencias docentes, el perfil del nuevo docente. Caracas, Venezuela. Recuperado de: https://books.google.co.ve/books?id=s1V7CAAAQBAJ

Castan, A. (2008). Material informático y contaminación medioambiental. Universidad de Argentina. Recuperado de: http://www.xtec.cat/ acastan/textos/Contaminacion\%20y\%20material \%20informatico.pdf

Hernández, R., Fernández, C., y Baptista, P. (2006). Metodología de la Investigación. 4ta edición. México. Recuperado de:

https://es.scribd.com/doc/38757804/Metodologia-de-La-InvestigacionHernandez-Fernandez-Batista-4ta-Edicion

Hidalgo, L. (2013). La Basura Electrónica y la Contaminación Ambiental. Quito Ecuador. Recuperado de: http://www.ute.edu.ec/fci/hidalgo.pdf 
Hurtado de Barrera, J. (2010). Metodología de la Investigación Holística Guía para la compresión holística de la ciencia. Cuarta Edición. Quirón Ediciones S.A. Cooperativa Editorial Magisterio (Bogotá, Colombia) Ciea-Sypal (Caracas, Venezuela).

Ley de Infogobierno (2013). Capítulo II. Principios y bases del uso de las tecnologías de información, Obligatoriedad del uso de las tecnologías de información. Venezuela. Recuperado de:

http://www.mpppst.gob.ve/mpppst/wpcontent/uploads/2015/02/LEY DE INFOGOBIERNO.pdf

Martínez, F., Naranjo, F., y Garrido, P. (2007). Reciclajes de equipos informáticos. XIII Jornada de enseñanza universitaria de la informática. Teruel, España.

Márquez, O. (2000). El proceso de Investigación de las ciencias sociales. UNELLEZ, Barinas, Venezuela.

Pineda, D. (2012). Modelo para la Gestión de Reciclaje de Residuos Electrónicos. Chile: Facultad de Ciencias Físicas y Matemáticas Departamento de Ingeniería Eléctrica. Recuperado de: http://www.repositorio.uchile.cl/bitstream/handle/2250/112257/cfpineda do.pdf?sequence $=1$

Sampieri, H. (2006). Metodología de la investigación. 4ta edición. México. UNESCO (2010). Los Residuos Electrónicos: Un desafío para la sociedad del conocimiento en América Latina y el Caribe (RELAC). [Documento en línea]. Recuperado de:

http://www.unesco.org.uy/ci/fileadmin/comunicacioninformacion/LibroE-Basura-web.pdf

UPEL (2014). Manual de Trabajo de Grado Especialización y Maestría y Tesis Doctorales. 4ta edición. Caracas, Venezuela. 


\section{Heiddy Ludmila Lizcano de Aviles \\ e-mail: kemyleo@gmail.com}

Nacida en Barinas, Municipio Barinas, Venezuela. Cursó estudios de primaria en la Escuela Básica Doña Menca de Leoni, Barinas, la secundaria en el Instituto Barinas recibiendo el título de bachiller en ciencias, curso Pregrado en la UNELLEZ VPDS obteniendo el título de Licenciada en Educación Mención Geografía e Historia, actualmente cursante de Maestría en Educación Ambiente y Desarrollo, UPEL Barinas. En la actualidad se desempeña como Docente Universitario UNELLEZ VPDS, adscrita al programa de ciencias sociales y jurídicas, subprograma de sociología del desarrollo, cumplo funciones de docencia, investigación y extensión bajos los perfiles de geografía, historia y ambiente, la formación se ha inclinado en la participación en Congresos Internacionales en Educación y Sociedad para La Emancipación M.E.P.E. CONSTRUNELLEZ, IMCLLANO, formación de Movimientos Sociales del Estado Barinas, formación de Tutores Para Trabajo de Grado del Programa Ciencias de la Educación UNELLEZ, ha actuado como ponente en actividades de Desafío de la Gerencia Educativa en el siglo XXI, en el Congresillo de Educación Universitaria: Miradas Criticas Por la Transformación Universitaria, Congreso Internacional de Investigación en Currículo y Formación Docente UNELLEZ, Barinas entre otras actividades relevante a la educación universitaria.

El contenido de este manuscrito se difunde bajo una Licencia de Creative Commons ReconocimientoNoComercial-Compartirlgual 4.0 Internacional 